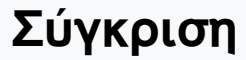

Tóp. 9 (1998)

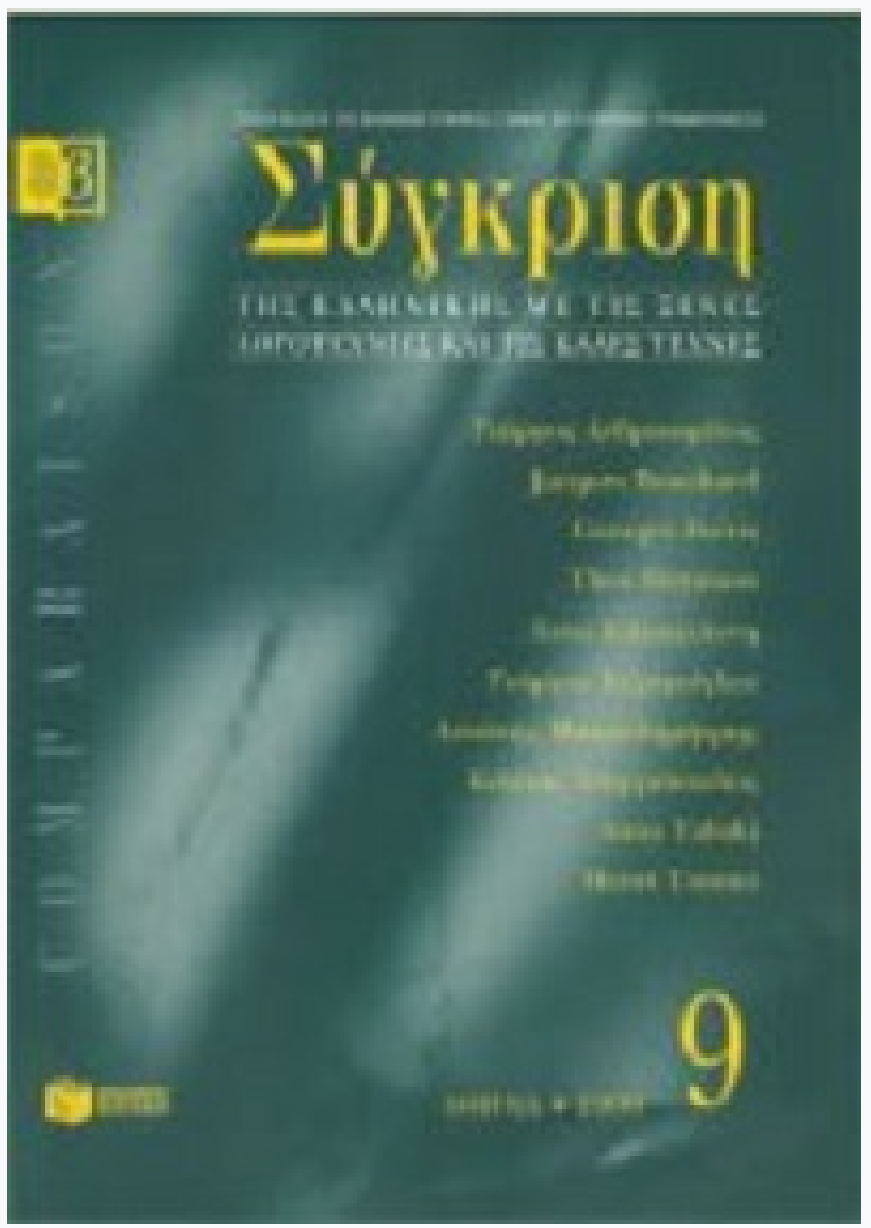

\section{Embiricos Lecteur de Proust}

Jacques Bouchard

doi: $10.12681 /$ comparison.11447

\section{Copyright $@$ 2017, Pavlos Matessis}

\section{(@) $\odot \Theta(\odot$}

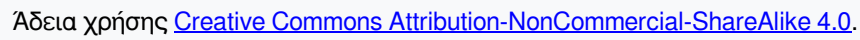

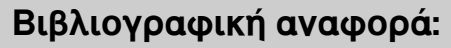

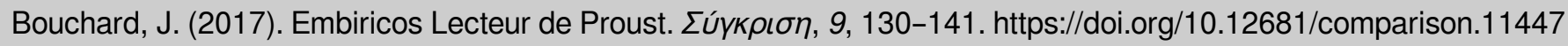




\section{Embiricos Lecteur de Proust*}

\section{à Pavlos Matessis}

$\mathrm{L}$ orsqu'un poète ou un prosateur prend place dans le trésor d'une littérature, la critique se doit d'expliciter les forces vives de son œuvre, de mettre en valeur l'originalité de son style et de situer cet apport unique parmi la production de son temps.

L'histoire de la littérature, comme celle de la peinture, ou de tout autre art, nous illustre abondamment la période de formation du créateur, celle pendant laquelle un peintre reproduit les grands maîtres en les copiant, un écrivain se repaît de lectures en assimilant des mots, des tropes, des styles. L'identification des sources n'expliquera jamais le mystère de la création, mais permettra au connaisseur d'apprécier l'écart entre le point de départ et le résultat obtenu, la création originale.

$\mathrm{Si}$ d'ordinaire, la critique procède par déductions, par recoupements qui visent à pallier le manque de sources sûres, dans sa patiente reconstitution de la genèse d'une ouvre, pour certains cas privilégiés, on dispose, en partant, de preuves matérielles irréfutables, dont l'exploitation lève en partie le voile sur la formation d'une conscience - et surtout d'une inconscience - littéraire.

Le cas qui nous occupera aujourd'hui est celui du poète, prosateur et psychanalyste Andréas Embiricos. Il a lui-même livré le nom de beaucoup de prosateurs ou de poètes qu'il estimait ou qui l'ont influencé. Qu'on pense,

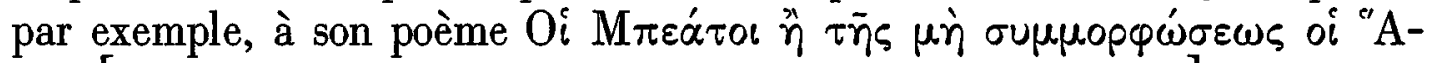
y versions sont connues. ${ }^{1}$. Dans ce texte on trouve mentionné le poète belge Henri Michaux, l'auteur du recueil L'Espace du dedans, publié en 1944:

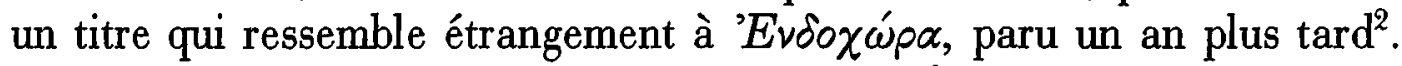

Par contre, à ma connaissance, Embiricos n'a jamais mentionné dans ses poèmes ou dans sa prose le nom de Marcel Proust; son roman ' $O M \mathcal{\varepsilon}^{\prime}-$

* Texte d'une communication lue le 19 mai 1994 au Colloque international «Surréalisme européen et surréalisme grec» tenu à Athènes, à la Fondation Goulandris-Horn. 


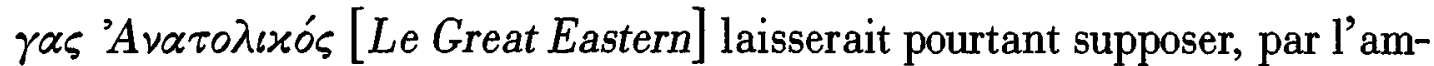
pleur de son développement, que l'auteur ait eu l'ambition de rivaliser avec le romancier français, dans sa fresque d'un microcosme, stylisée avec l'in-

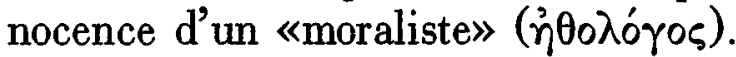

Mais avant d'entrer dans le vif du sujet, qu'il me soit permis de remercier ici publiquement madame Vivica Embiricos et monsieur Léonidas Embiricos de l'empressement et de la générosité avec lesquels ils ont mis à ma disposition la bibliothèque de l'écrivain, une immense bibliothèque, générale et spécialisée, qui mériterait une étude exhaustive et réserve sûrement d'autres surprises.

En examinant le contenu d'une des bibliothèques Embiricos, j'ai pu constater que neuf volumes des œuvres de Marcel Proust y sont conservés. Ils portent des marques qui intéressent indubitablement les études littéraires, et peut-être même l'histoire de la psychanalyse en Grèce.

Il s'agit de neuf volumes reliés mi-cuir avec titre or, des œuvres de Proust publiées chez Gallimard. Six d'entre eux portent la signature de l'acquéreur, le lieu et la date de l'acquisition. Ils ont tous été achetés à Nice, en 1926. Sur la page titre, l'acquéreur signe à l'anglaise: Andrew L. Embiricos. Le premier achat s'est fait le 17 mai 1926. Je me propose de décrire brièvement le titre des volumes en ajoutant quelques détails susceptibles d'intérêt; je m'attarderai ensuite sur les remarques personnelles d'Embiricos dont certains volumes gardent la trace.

1. Les plaisirs et les jours. La page de titre porte la mention: Andrew L. Embiricos, Nice 17/5/26. Embiricos possède la $26 \mathrm{e}$ édition, dont l'achevé d'imprimer date du 20 décembre 1924. Sur cet exemplaire, il n'y a ni commentaire manuscrit, ni soulignement; seul un petit trait devant certains intitulés de la page faisant face à la page titre: devant Les Plaisirs, puis devant tous les titres de $\grave{A}$ la recherche du temps perdu, sauf devant Albertine disparue. Il n'y a pas de marque devant les Pastiches et mélanges. Ces «obèles» indiquent qu'Embiricos avait l'intention d'acquérir les volumes en question, ou bien que l'acquisition en a été réalisée.

2. À l'ombre des jeunes filles en fleurs. Il s'agit du premier volume sous ce titre, marqué d'un astérisque (*), mais le tome II de $\grave{A}$ la recherche du temps perdu. Même mention de l'acquéreur, du lieu et de la date. Embiricos possède la 89e édition, de 1925. Cet exemplaire comporte de nombreux passages soulignés, de même que de nombreux traits verticaux dans la marge, accompagnés la plupart du temps d'un signe en forme de M majuscule (= Magnifique?), marquant l'intérêt du lecteur. Par exemple, Embiricos, polyglotte averti, signale d'un trait la remarque suivante, page 
143: «Dans une langue que nous savons, nous avons substitué à l'opacité des sons la transparence des idées. Mais une langue que nous ne savons pas est un palais clos dans lequel celle que nous aimons peut nous tromper, sans que, restés au dehors et désespérément crispés dans notre impuissance, nous parvenions à rien voir, à rien empêcher.»

Un peu plus bas, page 150, Embiricos manifeste son approbation à l'affirmation suivante en la soulignant: «Nous sommes tous obligés, pour rendre la réalité supportable, d'entretenir en nous quelques petites folies.»

Puis, à la page 197 de cette édition, une remarque pénétrante de Proust retient l'attention d'Embiricos: «Mais en tout genre, notre temps a la manie de vouloir ne montrer les choses qu'avec ce qui les entoure dans la réalité, et par là de supprimer l'essentiel, l'acte de l'esprit qui les isola d'elle.» Il

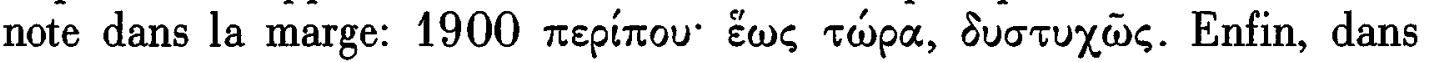
une page où il est question de «corps conscient», page 216, Embiricos souligne cette dernière phrase: «C'est notre attention qui met des objets dans une chambre, et l'habitude qui les en retire et nous y fait de la place.»

3. À l'ombre des jeunes filles en fleurs. Volume second, marqué de deux astérisques $(* *)$. La mention de l'acquéreur a été rognée par le relieur; même lieu et même date. C'est aussi la $89 \mathrm{e}$ édition. Cet exemplaire ne contient pas de commentaires, mais de nombreux traits verticaux en marge accompagnés du signe $\mathrm{M}$ majuscule. On y remarque un seul soulignement, page 196, concernant la finesse des mains féminines.

Malgré la rareté donc de notes marginales, on constate que les deux volumes de ce roman ont fait l'objet d'une lecture attentive d'Embiricos.

4. Le volume suivant contient Le côté de Guermantes II et Sodome et Gomorrhe I. 27e édition, 1921. Le nom de l'acquéreur est rogné par le relieur; même lieu et même date d'acquisition. Cet exemplaire ne comporte aucun commentaire, aucun soulignement; on ne peut donc affirmer qu'Embiricos a lu ce livre. Remarquons qu'il manque la première partie de Le côté de Guermantes dans l'état actuel de la collection Embiricos.

5. Embiricos possède aussi le roman Sodome et Gomorrhe II **, dans la 42e édition, 1924. Cet exemplaire est immaculé.

6. Même constatation concernant Sodome et Gomorrhe II ***, dans la 48e édition, 1924. Aucun commentaire, aucun soulignement.

7. Même remarque concernant La Prisonnière (Sodome et Gomorrhe III). Embiricos possède la 32e édition, 1924. Sauf la mention de l'acquéreur, du lieu et de la date d'acquisition en page titre, on n'y trouve aucun commentaire, ni soulignement.

8. \& 9. Mais les deux exemplaires de loin les plus intéressants, qui 
nous apprennent quelque chose sur l'écrivain et le psychanalyste Embiricos, ce sont les deux volumes du roman $D u$ côté de chez $S$ wann, formant le tome I de $\grave{A}$ la recherche du temps perdu. Embiricos a acquis les deux volumes dans leur 71e édition, dont l'achevé d'imprimer est daté du 24 juin 1926. Le premier volume porte la mention: Andrew L. Embiricos, Nice 27/8/1926. Ils ont donc été achetés trois mois après les 7 livres dont j’ai parlé précédemment. Le premier volume comporte de nombreux commentaires en français et en grec, de nombreux soulignements, des traits verticaux, parfois accompagnés du M majuscule ou d'un autre signe en forme d'alpha minuscule. Le second volume, lui, n'a pas d'ex-libris, ni de commentaires, mais contient de nombreux soulignés et traits verticaux.

Commençons par le premier volume. Les quelque 300 pages de cet exemplaire sont couverts de brefs commentaires, de renvois, de soulignés et de signes. Je choisirai les soulignements les plus intéressants, mais je vais tenter de vous donner une idée assez fidèle des commentaires. Ainsi, page 147, le narrateur dit de Bergotte et de Mlle Swann qu' «il cheminait à côté d'elle, inconnu et glorieux, comme les Dieux qui descen daient au milieu des mortels.» Embiricos entoure l'expression «inconnu et glorieux» et ajoute dans la marge $\pi \varepsilon p i \varepsilon p \gamma \eta ~ \varphi p a \sigma o u ́ \lambda \alpha$. Page 149, en marge d'une

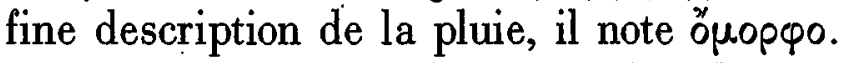

Page 165, là où il est question de M. Vinteuil et de son morceau de

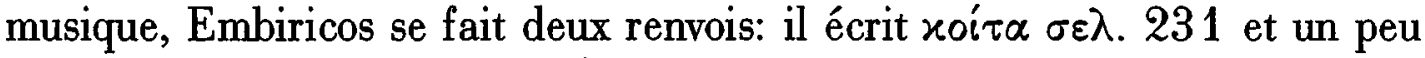
plus bas de la même page xoí $\alpha \sigma \varepsilon \lambda$. 233. De même, aux pages 231 et 233 , où le narrateur mentionne le portrait de M. Vinteuil, on trouve en marge жoí $\tau \alpha \sigma \varepsilon \lambda .165$.

Embiricos manifeste un grand intérêt pour l'analyse du snobisme faite par Marcel Proust: en haut de la page 181, il titre «Mr Legrandin», et page 187 en haut, comme à la page 191, il inscrit le mot «Snobisme». À la page 187, Embiricos exulte à la lecture de la description de Legrandin et souligne l'expression «comme un saint Sébastien du snobisme». Dans la marge, il écrit $\theta \alpha \zeta \mu \alpha ́ \sigma ı !$ avec un $\mathbf{b}$, ce qui - à première vue - étonne. Mais cette graphie corrobore une déclaration du poète, faite le 22 mai 1973, à l'Université de Thessalonique:

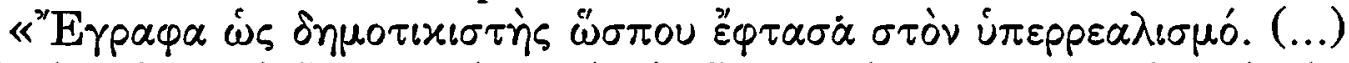

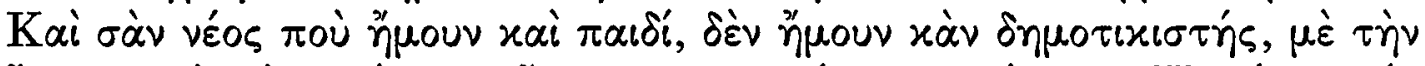

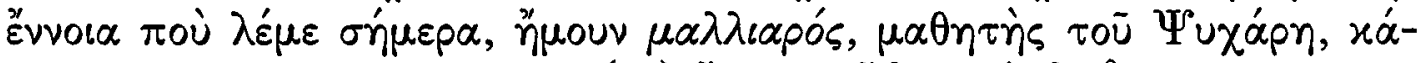

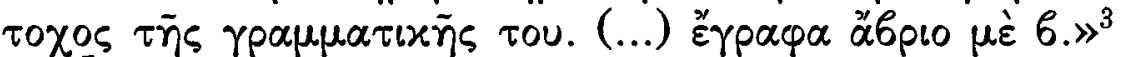

[J'écrivais en démotique avant d'accéder au surréalisme. (...) Dans mon jeune temps, je n'étais pas démoticiste au sens où on le dit aujourd'hui; j'é- 
tais un «poilu», un disciple de Psichari, un adepte de sa grammaire. (...) J'écrivais $\not$ $b$ pıo avec un 6 .]

Plus loin, Embiricos s'intéresse au personnage de Gilberte, dont il écrit le nom page 205, deux fois, puis page 206, avec la mention: «Gilberte Amour». Il souligne en outre le passage suivant: «Ainsi passa près de moi ce nom de Gilberte, donné comme un talisman qui me permettrait peutêtre...»

Page 223, un trait vertical rehausse la longue remarque de Proust qui va de «Quand j'étais fatigué d'avoir lu toute la matinée dans la salle»... jusqu'à «pour avoir préféré à un lent et difficile éclaircissement, le plaisir d'une dérivation plus aisée vers une issue immédiate». Embiricos marque ce passage d'un alpha dans la marge $(\alpha=$ de première importance?) et

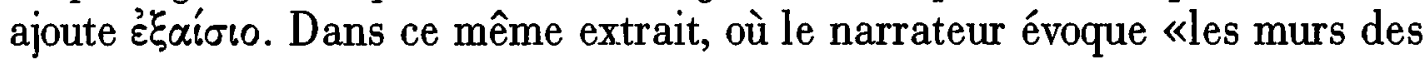
maisons, la haie de Tansonville, les arbres du bois de Roussainville, les buissons auxquels s'adosse Montjouvain», Embiricos souligne les mots qui suivent: [ils] «recevaient des coups de parapluie ou de canne, entendaient des cris joyeux, qui n'étaient, les uns et les autres, que des idées confuses qui m'exaltaient».

Toute la page 224, une description du paysage de Montjouvain après

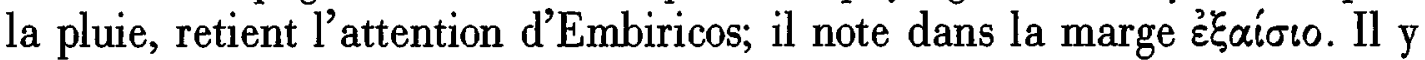
remarque aussi le passage fameux: «Et voyant sur l'eau et à la face du mur un pâle sourire répondre au sourire du ciel, je m'écriai dans mon enthousiasme en brandissant mon parapluie refermé: "/Zut, zut, zut, zut."/ Mais en même temps je sentis que mon devoir eût été de ne pas m'en tenir à ces propos opaques et de tâcher de voir plus clair dans mon ravissement.» Il souligne l'interjection répétée et ajoute un alpha dans la marge.

Embiricos titre la page 225 des mots «Le désir». Dans cette page il souligne la phrase suivante: «Parfois à l'exaltation que me donnait la solitude, s'en ajoutait une autre que je ne savais pas en départager nettement, causée par le désir de voir surgir devant moi une paysanne, que je pourrais serrer dans mes bras.» Notre lecteur ajoute une flèche $(\leftarrow)$, un $\mathrm{M}$ majuscule et un alpha minuscule dans la marge. Cette page est marquée d'un double trait vertical. Embiricos trace un trait vertical continu tout le long des pages suivantes, 226 et 227. Page 226, Proust écrit: «et mon imagination reprenant des forces au contact de ma sensualité, ma sensualité se répandant dans tous les domaines de mon imagination, mon désir n'avait plus de limites.» À la page 227, le narrateur se met à errer dans les bois de Roussainville à la recherche d'une paysanne à embrasser. Puis il ajoute: «Gette fille que je ne voyais que criblée de feuillages, elle était 
elle-même pour moi comme une plante locale d'une espèce plus élevée seulement que les autres et [à partir d'ici Embiricos souligne] dont la structure permet d'approcher de plus près qu'en elles, la saveur profonde du pays.» Embiricos ajoute un alpha dans la marge.

En haut de la page 229, Embiricos a écrit «M1l\{e\} Vinteuil et son ami». Un peu plus bas, Embiricos souligne le mot «sadisme» et note dans la marge: «Mlle Vinteuil et le sadisme». Page 233, en haut, Embiricos a écrit: «Mll $\{e\}$ Vinteuil et son amie». Un peu plus bas, àla phrase «Dans l'échancrure de son corsage de crêpe Mlle Vinteuil sentit que [et Embiricos souligne] son amie piquait un baiser», dans la marge, il commente: $\chi \alpha \rho-$

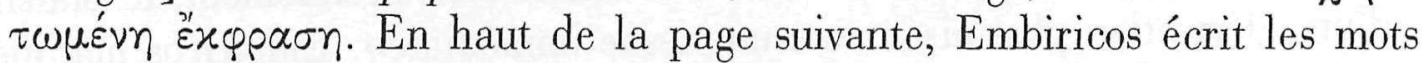
«Sur le sadisme». Toute la page est marquée d'un trait vertical. Sur une parenthèse du narrateur concernant la psychologie de Mlle Vinteuil, Embiricos écrit «l'indignation». Les pages 235 et 236 sont coiffées des mots d'Embiricos «Sur le sadisme». Vers la fin de la page 235, Embiricos souligne les mots «et il n'y a guère que le sadisme qui donne un fondement dans la vie à l'esthétique du mélodrame. Dans la réalité, en dehors des cas de sadisme...»D Dans la marge il a marqué un $\mathbf{S}$ majuscule (probablement un sigle pour $\Sigma \alpha \delta i \sigma \mu o ́ s)$. La page 236 porte des soulignés, un M majuscule, un alpha minuscule et un $\mathbf{S}$ majuscule. Deux M majuscules et deux alphas minuscules signalent l'intérêt de la page 237, coiffée des mots «Analyse de Mlle Vinteuil». Au bas de la page, on lit l'appréciation enthousiaste d'Embiricos «Analyse $\mathrm{p}[\mathrm{h}]$ sychologique magistrale».

Les pages 250 et 251 portent les mots «Mme de Guermantes». En haut de la page 255, Embiricos a écrit «Mme de Guermantes. Amour». Dans la marge, à la hauteur de la phrase «Alors me rappelant ce regard qu'elle avait laissé s'arrêter sur moi, pendant la messe, bleu comme un rayon de soleil qui aurait traversé le vitrail de Gilbert le Mauvais», Embiricos commente d'un mot: öpopழo. Il marque d'un alpha dans la marge la phrase; «Et aussitôt je l'aimai, car s'il peut quelquefois suffire pour que nous aimions une femme (souligné jusqu'ici par Embiricos) qu' elle nous regarde avec mépris comme j’avais cru qu' avait fait Mlle Swann et que nous pensions qu'elle ne pourra jamais nous appartenir, quelquefois aussi il peut suffire qu'elle nous regarde avec bonté comme faisait Mme de Guermantes et que nous pensions qu'elle pourra nous appartenir.» De la page suivante, il souligne l'expression «un épiderme de lumière»; mais la page presque entière est marquée des alpha minuscule et $\mathrm{M}$ majuscule.

En haut des pages 272 et 273 , Embiricos a écrit «Les Verdurin[s]»; en marge de la première ligne, soulignée, de la page 272 «d'une 
respectable famille bourgeoise excessivement», Embiricos note «Mme Verdurin».

Les dernières annotations d'Embiricos concernent Swann: en haut de la page 275 , il écrit «Swann et les femmes» et il souligne plus bas la phrase «Swann aimait tellement les femmes»; en haut de la page 276 , il note «Swann. Vanité - Amour»; en haut de la page suivante «Swann et sa façon d'agir en amour». Dans cette page, il souligne presque tout le premier paragraphe: «Et c'étaient souvent des femmes de beauté assez vulgaire, car les qualités physiques qu'il recherchait sans s'en rendre compte étaient en complète opposition avec celles qui lui rendaient admirables les femmes sculptées ou peintes par les maîtres qu’il préférait. La profondeur, la mélancolie de l'expression, glaçaient ses sens que suffisait au contraire à éveiller une chair saine, plantureuse et rose.»

Enfin, Embiricos marque les pages 296, 297 et 298, d'un M majuscule, tout en soulignant au bas de la page 297 et en haut de la page suivante la phrase: «Peut-être aussi à force de dire qu'elle serait malade, y. avait-il des moments où elle ne se rappelait plus que c'était un mensonge et [298] prenait une âme de malade.» C'est là le dernier souligné du livre.

Le second volume du rọman Du côté de chez $S$ wann ne comporte pas de commentaires marginaux de la main d'Embiricos. On y constate cependant qu'il l'a lu attentivement, le crayon à la main; il a couvert son exemplaire de soulignements et de traits verticaux, parfois doubles, en général accompagnés du M majuscule. On y trouve soulignées ou marquées d'un $M$ des réflexions de Proust sur le désir, des analyses de sentiments et de sensations, et aussi des descriptions de forêts.

Mentionnons à titre d'exemple qu'Embiricos souligne la dernière ligne de la page 249 et les deux premières de la page 250 où se lit la pensée suivante: «Il y a des jours montueux et malaisés qu'on met un [250] temps infini à gravir et des jours en pente qui se laissent descendre à fond de train en chantant.»

Enfin, les dernières pages, de 292 à 301 , sont marquées d'un trait vertical, - double aux pages 292 à 296 - , où Embiricos remarque une magnifique évocation du Bois de Boulogne, qui commence comme suit: «Cette complexité du bois de Boulogne qui en fait un lieu factice et, dans le sens zoologique ou mythologique du mot, un Jardin, je l'ai retrouvée cette année comme je le traversais pour aller à Trianon...» La prose de Marcel Proust chante comme de la poésie imprégnée de couleurs et d'allitérations: «Plus loin, là où toutes les feuilles vertes couvraient les arbres, un seul, petit, trapu, étêté et têtu, secouait au vent une vilaine chevelure rouge.» (page 
293). À la page 294, on lit: «Et c'était aussi l'heure. Dans les endroits où les arbres gardaient encore leurs feuilles, ils semblaient subir une altération de leur matière à partir du point où ils étaient touchés par la lumière du soleil, presque horizontalement le matin comme elle le redeviendrait quelques heures plus tard au moment où dans le crépuscule commençant, elle s'allume comme une lampe, projette à distance sur le feuillage un reflet artificiel et chaud...» Un peu plus bas, page 295, Proust esquisse les différentes parties du Bois en ces termes: «Des espaces plus éclaircis laissaient voir l'entrée de presque toutes, ou bien un feuillage somptueux la désignait comme une oriflamme.» [... «On sentait que le Bois n'était pas qu'un bois, qu'il répondait à une destination étrangère à la vie des arbres, l'exaltation que j'éprouvais n'était pas causée que par l'admiration de l'automne, mais par un désir. Grande source d'une joie que l'âme ressent d'abord sans en connaître la cause, sans comprendre que rien au dehors ne la motive. Aussi regardais-je les arbres avec une tendresse insatisfaite qui les dépassait et se portait à mon insu vers ce chef-d'œuvre des belles promeneuses qu'ils enferment chaque jour pendant quelques heures.»

Personnellement, il me semble que quelque chose de ces pages se retrouve dans certaines évocations de parcs ou de jardins, chez Embiricos. Par exemple, dans son poème Oi Kapuá

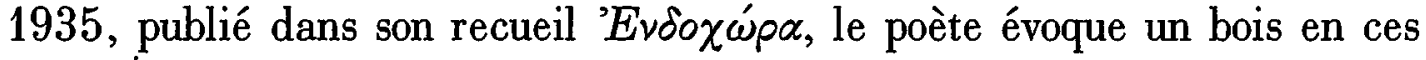
termes ${ }^{1}$ :

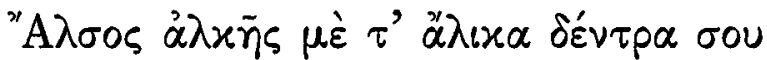

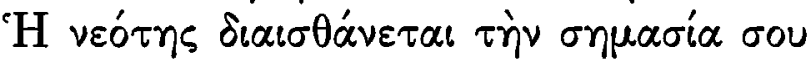

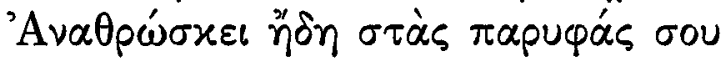

[Bosquet de verdeur avec tes arbres écarlates

La jeunesse par intuition saisit ta signification

Depuis déjà tes lisières elle se laisse percevoir]

et plus bas, à propos des jeunes filles:

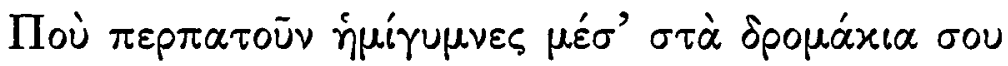

[Qui déambulent à moitié nues dans tes sentiers]

Et la fin majestueuse:

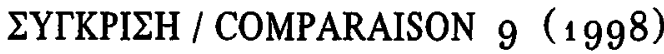




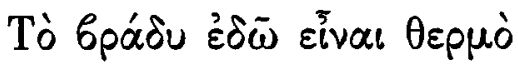

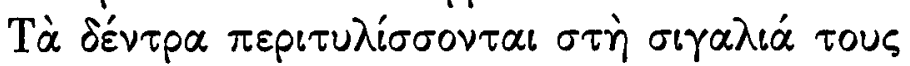

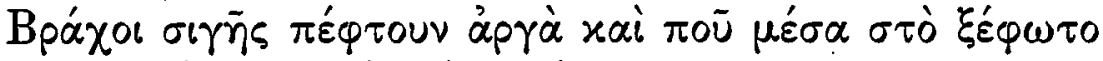

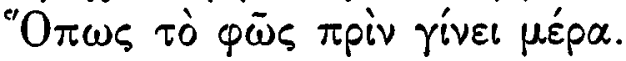

[Ici la soirée sera chaude

Les arbres se couvrent de leur sérénité

Des blocs de silence s'écroulent par intervalles dans la clairière Comme la lumière avant qu'il fasse jour.]

Du second volume du roman $D u$ côté de chez Swann, Embiricos a souligné les dernières lignes de la page 301, par lesquelles Proust terminait son livre: «le souvenir d'une certaine image n'est que le regret d'un certain instant; et les maisons, les routes, les avenues, sont fugitives, hélas, comme les années.»

Outre les 9 volumes que je viens de mentionner, la bibliothèque d'Andréas Embiricos en conserve 4 autres qui prouvent éloquemment le vif intérêt du lecteur grec pour le romancier français. Il s'agit d'abord de l'Essai sur Marcel Proust de Georges Gabory, publié chez Émile Chamontin en 1926. Ce livre porte la mention: Andrew L. Embiricos, Paris 11/6/1926. On y trouve encore une monographie de Léon Pierre-Quint, sans ex-libris, mais qu'Embiricos a dû se procurer à la même époque, intitulée Marcel Proust, sa vie, son auvre, parue aux Éditions du Sagittaire, en 1925. En outre, Embiricos possède l'ouvrage de Robert Dreyfus, Souvenirs sur Marcel Proust, avec des lettres inédites de Marcel Proust, paru chez Grasset en 1926. Ce livre porte la mention: Andrew L. Embiricos, Nice 5/1/1927. Ces trois volumes sont reliés de la même manière que les ouvres de Proust. Enfin la bibliothèque Embiricos conserve un exemplaire, non relié, du livre d'André Germain, De Proust à Dada, publié aux Éditions du Sagittaire en 1924. On y trouve la mention: Andrew L. Embiricos, Nice 18/5/1926.

Sans rien présumer de certain concernant la date à laquelle Andréas Embiricos a pu lire Marcel Proust, j'émettrai l'hypothèse qu'il a dû lire de ses romans entre 1926 et 1928, vu la nature de ses annotations, plus psychologiques et esthétiques que psychanalytiques et littéraires. De plus l'orthographe de $\theta a b \mu a ́ \sigma t o$ avec un $\boldsymbol{b}$ nous indiquerait qu'il a lu Proust avant son adhésion au surréalisme. Car, c'est alors qu'Embiricos est revenu à la langue qui lui était «naturelle», - puisqu'il avait reçu toute son éducation en langue savante, - c'est-à-dire qu'il est revenu à la langue «mixte», comme il le précise dans sa déclaration de 1973 à l'Université de Thes- 
salonique $^{5}$. Or c'est vraisemblablement en 1929 que le psychanalyste Frois Wittmann a présenté Embiricos à André Breton ${ }^{6}$.

Il semble qu'Embiricos ait délaissé par la suite l'œuvre proustienne, sûrement influencé en cela par le discrédit général manifesté par les surréalistes à l'égard de Proust ${ }^{7}$; quoi qu'il en soit, il n'est apparemment pas fait mention de Proust chez Embiricos. Il est pourtant loisible de penser qu'une lecture aussi attentive de ce grand écrivain par le jeune Andréas a dû laisser des traces profondes dans la sensibilité littéraire et professionnelle d'un Embiricos plus mûr.

Je suis persuadé qu'une étude philologique méthodique prouverait certaines réminiscences des écrits de Proust dans l'œuvre romanesque d'Embiricos, et même dans son œuvre poétique.

En guise d'exemple, je me bornerai, en terminant, à suggérer un parallèle possible entre la remarque de Proust concernant la duchesse de Guermantes, dont chaque robe était, écrit-il, «comme la projection d'un

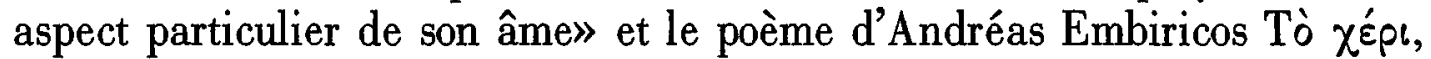

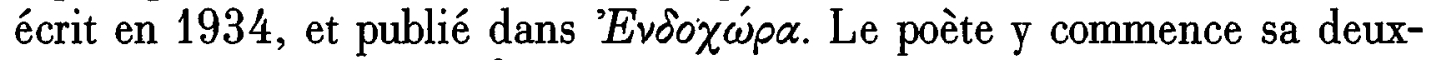
ième strophe par les vers ${ }^{8}$ :

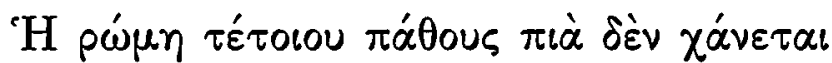

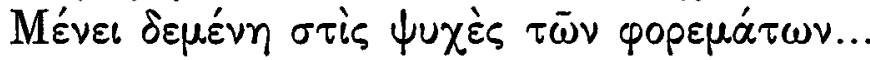

[La robustesse d'une telle passion ne passe plus

Elle reste imprégnée dans l'âme des vêtements]

\section{Notes}

1. A. Embiricos, 'Oxtáva, Athènes, Ikaros, 1980, p. 36-38 et 101-103.

2. H. Michaux a aussi publié Lointain intérieur en 1938. En fait la mention de

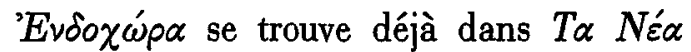
$\Gamma$ ра́ $\mu \mu \alpha \tau \alpha$ de mai 1937 , p. 345; les poèmes publiés par Embiricos y portent la

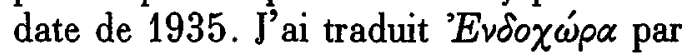
Terre intérieure dans mes précédentes publications pour éviter «Espace intérieur», trop évocateur de Michaux; je me rallie à Gilles Ortlieb qui propose Domaine intérieur, in Le nouveau diction- naire des auteurs, Paris, Laffont-Bompiani, vol. I, 1994, p. 999.

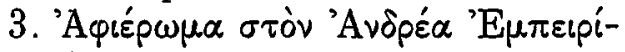

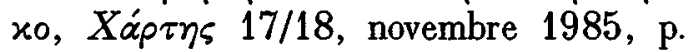
638.

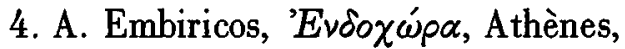
1945; réédition Agra, 1980, p. 75-76. Ma traduction de ce poème a été publiée dans

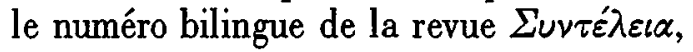
no 4-5, printemps-été 1991, p. 11. 639 .

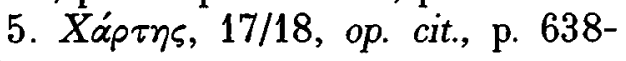

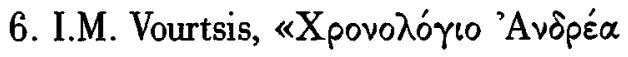




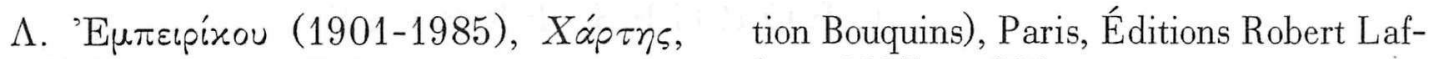
17/18, op. cit., p. 518 .

7. Voir l'introduction de Philippe Michel-Thériet dans: Marcel Proust, A la

recherche du temps perdu, vol. I, (Collecfont, 1987 , p. 235.

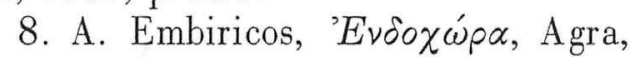
1980, p. 38. Ma traduction dans $\Sigma u \nu \tau \varepsilon ́-$ - $\lambda \varepsilon \iota \alpha$, no 4-5, printemps-été 1991 , p. 9.

\section{$\Pi \varepsilon \rho i \lambda \eta \psi \eta$}

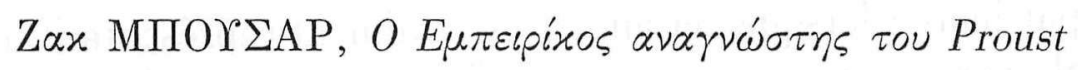

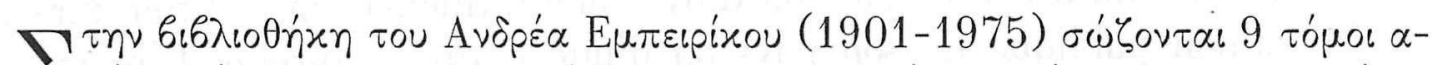

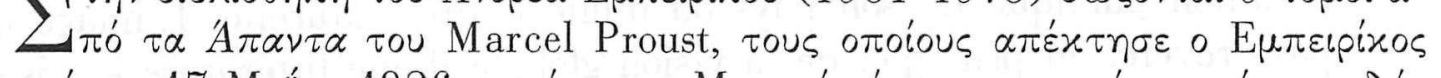

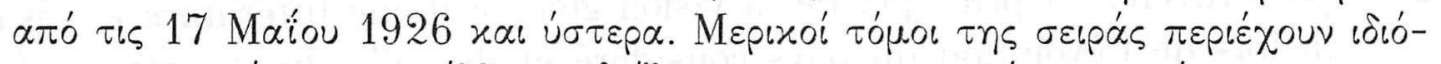

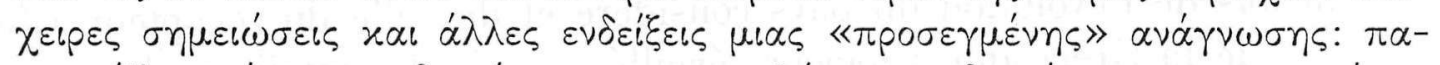

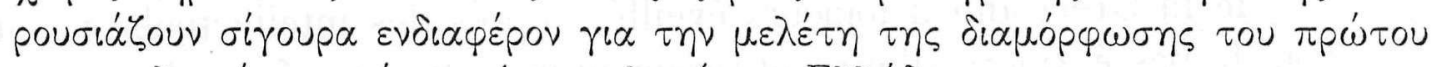

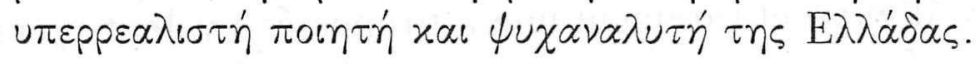

